\title{
Diblock copolymer/homopolymer blends: Derivation of a density functional theory
}

\author{
Rustum Choksi ${ }^{\mathrm{a}, *}$, Xiaofeng Ren ${ }^{\mathrm{b}}$ \\ a Department of Mathematics, Simon Fraser University, 8888 University Dr., Burnaby, BC, Canada V5A 1S6 \\ b Department of Mathematics, Utah State University, Logan, UT 84322-3900, USA
}

Received 20 October 2004; received in revised form 17 February 2005; accepted 11 March 2005

Communicated by Y. Nishiura

\begin{abstract}
Melts of diblock copolymer/homopolymer blends exhibit multiscale phase separation: (i) macrophase separation into homopolymer- and copolymer-rich macrodomains followed by (ii) microphase separation into A- and B-rich microdomains within the copolymer-rich macrodomains (cf. [S. Koizumi, H. Hasegawa, T. Hashimoto, Macromolecules 27 (1994) 6532; S. Koizumi, H. Hasegawa, T. Hashimoto, Macromolecules 27 (1994) 7893; H. Tanaka, H. Hasegawa, T. Hashimoto, Macromolecules 24 (1991) 240]). Following our previous derivation in [R. Choksi, X. Ren, On a derivation of a density functional theory for microphase separation of diblock copolymers, J. Stat. Phys. 113 (2003) 151-176], we derive a density functional theory for blends. This theory has been shown numerically to capture the multiscale separation (cf. [T. Ohta, A. Ito, Dynamics of phase separation in copolymer-homopolymer mixtures, Phys. Rev. E 52-5 (1995) 5250-5260; A. Ito, Domain patterns in copolymer-homopolymer mixtures, Phys. Rev. E 58-5 (1998) 6158-6165]). We also prove a result on local minimizers in one space dimension, confirming a lamellar multiscale phase separation.
\end{abstract}

(C) 2004 Elsevier B.V. All rights reserved.

PACS: $61.25 . \mathrm{Hq} ; 61.43 . \mathrm{Bn} ; 64.70$

Keywords: Multiscale phase separation; Diblock copolymer/homopolymer blend; Density functional theory

\section{Introduction}

A diblock copolymer is a linear-chain molecule consisting of two sub-chains joined covalently to each other. One of the sub-chains is made of monomers of type A and the other of type B. Below a critical temperature, even a weak repulsion between unlike monomers $\mathrm{A}$ and $\mathrm{B}$ induces a strong repulsion between the sub-chains, causing the sub-chains to segregate. A macroscopic segregation, whereby the sub-chains detach from one another, cannot occur

\footnotetext{
* Corresponding author. Tel.: +1 604291 3379; fax: +1 6042914947.

E-mail addresses: choksi@math.sfu.ca (R. Choksi), ren@math.usu.edu (X. Ren)
} 
because the sub-chains are chemically bonded: rather, a phase separation on a mesoscopic scale with A and B-rich domains emerges. The mesoscopic domains which are observed are highly regular periodic structures; for example, lamellar, bcc centered spheres, cylindrical tubes, and bicontinuous gyroids (see for example, [3] and the references therein). This phenomena of diblock copolymer melts is commonly referred to as microphase separation.

In this article, we consider a mixture of $\mathrm{A}-\mathrm{B}$ copolymers with homopolymers of monomer species $\mathrm{C}$, where the species $\mathrm{C}$ is thermodynamically incompatible with both the $\mathrm{A}$ and $\mathrm{B}$ monomer species. By a homopolymer of species $\mathrm{C}$ we mean a polymer chain consisting purely of the monomer species $\mathrm{C}$, and hence, a diblock copolymer results from covalently joining one end of a A-homopolymer to one end of a B-homopolymer. When such a mixture contains a sufficient concentration of the $\mathrm{C}$ homopolymers, the result in the melt phase is a macroscopic phase separation (which we call macrophase separation) into homopolymer- and copolymer-rich domains followed by microphase separation within the copolymer-rich domains into A- and B-rich subdomains (cf. [16,17,26]). Following this phenomena, let us agree to call the C-rich domains macrodomains and the A- and B-rich domains microdomains.

Following the work of Leibler [19], Ohta and Kawasaki [23] proposed a density functional theory (DFT) to model microphase separation of diblock copolymers wherein the energy of the system is written exclusively in terms of the averaged relative monomer density fields $u_{\mathrm{A}}$ and $u_{\mathrm{B}}$. This free energy entails a local Cahn-Hilliard-like (cf. [5,22]) term together with a nonlocal interaction term stemming from the connectivity of the chains.

Ohta and Ito $[24,18]$ later noted that the Ohta-Kawasaki DFT could be generalized to the case of blends. Using an appropriate gradient flow, they presented simulations on the dynamics of the micro-macro phase separation. In particular, working with two order parameters (see Appendix A of the present article) they wrote down a precise form of the nonlocal interaction term. No details were presented on its derivation and we are unaware of any systematic derivation of such a nonlocal functional for a homopolymer/copolymer blend.

In [6], we gave a systematic derivation of the Ohta-Kawasaki DFT for microphase separation of diblock copolymers, presenting it as an offspring of the the self-consistent mean field theory (cf. [20] and the references therein). The main purpose of this article is to derive a similar DFT for a homopolymer/copolymer blend in terms of the averaged relative monomer density fields $u_{\mathrm{A}}, u_{\mathrm{B}}$ and $u_{\mathrm{C}}$. Because of the local nature of the mean field approximation and the linear framework for the entropic part of the free energy, the addition of the homopolymers simply induces a linear perturbation of the pure diblock problem considered in [6]. While we do outline all the major steps, we detail only the modifications, and refer the reader to [6] for missing details. One simplification over the approach in [6] is used: By using periodic boundary conditions, we simplify several of the steps.

Finally, we include a separate section (Section 4) devoted to proving a result in one space dimension on the existence of local minimizers which display a multiscaled lamellar pattern.

\section{Notation}

For clarity, we provide a brief summary of some of our notation:

- $\Omega \subset \mathbf{R}^{3}$ is the physical domain with volume $|\Omega|$ on which the melt lives and $D$ is a normalized physical domain of unit volume. Unlike in [6], we will assume here that $\Omega$ and $D$ are the cubes $(0, L)^{3}$ and $(0,1)^{3}$ respectively, and work with periodic boundary conditions; that is, we identify $\Omega$ and $D$ with the three dimensional cubic torus $T^{3}$ of dimension $L$ and 1 , respectively. We use $x-z$ to denote points in $\Omega$ with $\mathrm{d} x$ (or $\mathrm{d} y, \mathrm{~d} z$ ) in reference to a volume integral.

- Fields with super and sub indices of A-C will be used to denote reference to the A-C monomers, respectively. In Section 3, we often use $k$ and $m$ as variables taking on the values of either $A$ and $B$ or $\mathrm{A}-\mathrm{C}$. To distinguish between sums over $\mathrm{A}$ and $\mathrm{B}$ and sums over $\mathrm{A}-\mathrm{C}$ we use explicit summation notation rather than the summation convention.

- The indices of polymerization for the copolymer and homopolymer chains are denoted respectively by the integers $N$ and $N_{\mathrm{C}}$. For a copolymer chains parametrized by either $\tau$ or $t \in[0, N]$, we let $\mathcal{I}_{\mathrm{A}}=\left[0, N_{\mathrm{A}}\right)$ denote 
the interval occupied by the A-monomers and $\mathcal{I}_{\mathrm{B}}=\left[N_{\mathrm{A}}, N\right]$ denote the interval occupied by the B-monomers. We let $N_{\mathrm{B}}:=N-N_{\mathrm{A}}$. The molecular weight of the $\mathrm{A}$ and B monomers are $N_{\mathrm{A}} / N$ and $N_{\mathrm{B}} / N$, respectively.

- $r^{\mathcal{D}}=\left(r_{1}^{\mathcal{D}}, \ldots, r_{n}^{\mathcal{D}}\right)$ denotes an $n$-tuple of copolymer chains (continuous functions from $[0, N]$ to $\Omega$ ) and $r^{\mathcal{H}}=$ $\left(r_{1}^{\mathcal{H}}, \ldots, r_{v}^{\mathcal{H}}\right)$ denotes an $v$-tuple of homopolymer chains (continuous functions from $\left[0, N_{\mathrm{C}}\right]$ to $\Omega$ ). Associated to these $(n+v)$-tuples is an $(n+v)$-product Wiener measure denoted by $\mathrm{d} \mu$ (see Section 3 for a precise definition).

- $l$ denotes the Kuhn statistical length. The Boltzmann constant has been normalized to unity and $\beta$ denotes the reciprocal of the absolute temperature measured in units of (energy $)^{-1}$.

\section{Mean field approximation}

We assume there are $n \mathrm{AB}$-diblocks chains written: $r_{1}^{\mathcal{D}}, \ldots r_{n}^{\mathcal{D}}$ and $v \mathrm{C}$-homopolymer chains written: $r_{1}^{\mathcal{H}}, \ldots r_{v}^{\mathcal{H}}$. Each of the diblock chains $r_{i}^{\mathcal{D}}$ is a Brownian process in the function space

$$
\Gamma_{i}^{\mathcal{D}}=C([0, N], \Omega)
$$

and each of the homopolymer chains $r_{i}^{\mathcal{H}}$ is a Brownian process in the function space

$$
\Gamma_{i}^{\mathcal{H}}=C\left(\left[0, N_{C}\right], \Omega\right)
$$

If we write every $r_{i}^{\mathcal{D}} \in C([0, N], \Omega)$ as $r_{i}^{\mathcal{D}}=r_{i}^{\mathcal{D}}(0)+\left(r_{i}^{\mathcal{D}}-r_{i}^{\mathcal{D}}(0)\right)$, the space is decomposed into

$$
C([0, N], \Omega) \equiv \Omega \times\left\{r_{i}^{\mathcal{D}} \in C([0, N], \Omega): r_{i}^{\mathcal{D}}(0)=\overrightarrow{0}\right\} .
$$

Let $\mathrm{d} P_{\overrightarrow{0}}^{\mathcal{D}}$ be the Wiener measure of the standard Brownian motion, scaled by a factor $l / \sqrt{3}$, on $\left\{r_{i}^{\mathcal{D}} \in C([0, N], \Omega)\right.$ : $\left.r_{i}^{\mathcal{D}}(0)=\overrightarrow{0}\right\} . l$ is the Kuhn statistical length [7,9] that measures the average distance between two adjacent monomers. In this paper we only consider the situation when this $l$ is independent of the types of the adjacent monomers.

This Wiener measure is often written formally as

$$
\mathrm{d} P_{\overrightarrow{0}}^{\mathcal{D}} \sim \exp \left(-\frac{3}{2 l^{2}} \int_{0}^{N}\left(\frac{\mathrm{d} r_{i}^{\mathcal{D}}(\tau)}{\mathrm{d} \tau}\right)^{2} \mathrm{~d} \tau\right) \mathrm{d} r_{i}^{\mathcal{D}}
$$

Then the space $C([0, N], \Omega)$ is equipped with the measure

$$
\mathrm{d} \mu_{1}^{\mathcal{D}}=\mathrm{d} x \times \mathrm{d} P_{\overrightarrow{0}}^{\mathcal{D}} .
$$

With $n$ chains in the material, we consider the space of diblocks is

$$
\Gamma^{\mathcal{D}}=\left\{r^{\mathcal{D}}=\left(r_{1}^{\mathcal{D}} \ldots, r_{n}^{\mathcal{D}}\right): r_{i}^{\mathcal{D}} \in C\left([0, N], \boldsymbol{R}^{3}\right)\right\}
$$

equipped with the product measure

$$
\mathrm{d} \mu^{\mathcal{D}}=\underbrace{\mathrm{d} \mu_{1}^{\mathcal{D}} \times \mathrm{d} \mu_{1}^{\mathcal{D}} \times \cdots \times \mathrm{d} \mu_{1}^{\mathcal{D}}}_{n} .
$$


The analogous definitions apply for the homopolymer chains defining $r^{\mathcal{H}}, \mathrm{d} P^{\mathcal{H}}, \mathrm{d} \mu_{1}^{\mathcal{H}}, \mathrm{d} \mu^{\mathcal{H}}$, and $\Gamma^{\mathcal{H}}$. Thus, our phase space is

$$
\Gamma=\Gamma^{\mathcal{D}} \times \Gamma^{\mathcal{H}}
$$

equipped with the full product measure $\mathrm{d} \mu=\mathrm{d} \mu^{\mathcal{D}} \times \mathrm{d} \mu^{\mathcal{H}}$.

For the diblocks, the $\mathrm{A}\left(\mathrm{B}\right.$, respectively) monomers occupy the interval $\mathcal{I}_{\mathrm{A}}=\left[0, N_{\mathrm{A}}\right)\left(\mathcal{I}_{\mathrm{B}}=\left[N_{\mathrm{A}}, N\right]\right.$ respectively).With $n$ and $v$ chains of polymerization indices $N$ and $N_{\mathrm{C}}$, respectively, there are $n N+v N_{\mathrm{C}}$ monomers. Inside $\Omega$, the total average monomer number density is

$$
\rho_{0}=\frac{n N+v N_{\mathrm{C}}}{|\Omega|}
$$

We also have the average monomer number densities for the diblocks and homopolymers

$$
\rho_{0}^{\mathcal{D}}=\frac{n N}{|\Omega|} \quad \rho_{0}^{\mathcal{H}}=\frac{v N_{\mathrm{C}}}{|\Omega|}
$$

Next we introduce the interaction Hamiltonian on $\Gamma$. Let $V^{\mathrm{AA}}, V^{\mathrm{BB}}, V^{\mathrm{CC}}, V^{\mathrm{AB}}\left(=V^{\mathrm{BA}}\right), V^{\mathrm{AC}}\left(=V^{\mathrm{CA}}\right)$, $V^{\mathrm{BC}}\left(=V^{\mathrm{CB}}\right)$ (all positive) denote the interaction parameters. The interaction Hamiltonian is now

$$
\begin{aligned}
H\left(r^{\mathcal{D}}, r^{\mathcal{H}}\right)= & \sum_{i, j} \sum_{\mathrm{k}, \mathrm{m}=\mathrm{A}, \mathrm{B}} \frac{V^{\mathrm{km}}}{2 \rho_{0}} \int_{\mathcal{I}_{\mathrm{k}}} \int_{\mathcal{I}_{\mathrm{m}}} \delta\left(r_{i}^{\mathcal{D}}(\tau)-r_{j}^{\mathcal{D}}(t)\right) \mathrm{d} \tau \mathrm{d} t \\
& +\sum_{i, j} \sum_{\mathrm{k}=\mathrm{A}, \mathrm{B}} \frac{V^{\mathrm{kC}}}{\rho_{0}} \int_{\mathcal{I}_{\mathrm{k}}} \int_{0}^{N_{\mathrm{C}}} \delta\left(r_{i}^{\mathcal{D}}(\tau)-r_{j}^{\mathcal{H}}(t)\right) \mathrm{d} \tau \mathrm{d} t \\
& +\sum_{i, j} \frac{V^{\mathrm{CC}}}{2 \rho_{0}} \int_{0}^{N_{\mathrm{C}}} \int_{0}^{N_{\mathrm{C}}} \delta\left(r_{i}^{\mathcal{H}}(\tau)-r_{j}^{\mathcal{H}}(t)\right) \mathrm{d} \tau \mathrm{d} t,
\end{aligned}
$$

where here, the indices $i, j$ go from $1, \ldots, n$ for $r_{i}^{\mathcal{D}}, r_{j}^{\mathcal{D}}$, and $1, \ldots, v$ for $r_{i}^{\mathcal{H}}, r_{j}^{\mathcal{H}}$, and the indices $\mathrm{k}$ and $\mathrm{m}$ take on the values of $\mathrm{A}$ and $\mathrm{B}$, respectively. Note that factor $1 / 2$ is missing in the second term to account for the missing $V^{\mathrm{Ck}}$ terms, and that we assume that the interaction is short ranged in the use of the $\delta$-function. With this in hand, we can now write the Gibbs canonical distribution and partition function:

$$
D\left(r^{\mathcal{D}}, r^{\mathcal{H}}\right)=\frac{1}{Z} \exp \left(-\beta H\left(r^{\mathcal{D}}, r^{\mathcal{H}}\right)\right), \quad Z=\int_{\Gamma} \exp \left(-\beta H\left(r^{\mathcal{D}}, r^{\mathcal{H}}\right)\right) \mathrm{d} \mu
$$

which describes the thermal equilibrium. Here we use the energy unit to measure the absolute temperature so the Boltzmann constant is 1 and $\beta$ is the reciprocal of the absolute temperature. The free energy of the system is $-\beta^{-1} \log Z$. Introducing the microscopic densities

$$
\rho_{\mathrm{k}}\left(x, r^{\mathcal{D}}\right)=\sum_{i=1}^{n} \int_{\mathcal{I}_{\mathrm{k}}} \delta\left(x-r_{i}^{\mathcal{D}}(\tau)\right) \mathrm{d} \tau, \quad \mathrm{k}=\mathrm{A} \text { and } \mathrm{B},
$$


and

$$
\rho_{\mathrm{C}}\left(x, r^{\mathcal{H}}\right)=\sum_{i=1}^{n} \int_{0}^{N_{\mathrm{C}}} \delta\left(x-r_{i}^{\mathcal{H}}(\tau)\right) \mathrm{d} \tau,
$$

we define the macroscopic densities:

$$
\left\langle\rho_{\mathrm{k}}(x)\right\rangle=\int_{\Gamma} \rho_{\mathrm{k}}\left(x, r^{\mathcal{D}}\right) D(r) d \mu, \quad \mathrm{k}=\mathrm{A} \text { and } \mathrm{B} . \quad\left\langle\rho_{\mathrm{C}}(x)\right\rangle=\int_{\Gamma} \rho_{\mathrm{C}}\left(x, r^{\mathcal{H}}\right) D(r) d \mu .
$$

In terms of these macroscopic densities, the Hamiltonian (3.3) can be written

$$
\begin{aligned}
H\left(r^{\mathcal{D}}, r^{\mathcal{H}}\right)= & \int_{\Omega} \sum_{\mathrm{k}, \mathrm{m}=\mathrm{A}, \mathrm{B}} \frac{V^{k m}}{2 \rho_{0}} \rho_{\mathrm{k}}\left(x, r^{\mathcal{D}}\right) \rho_{\mathrm{m}}\left(x, r^{\mathcal{D}}\right) \mathrm{d} x+\int_{\Omega} \sum_{\mathrm{k}=\mathrm{A}, \mathrm{B}} \frac{V^{\mathrm{kC}}}{\rho_{0}} \rho_{\mathrm{k}}\left(x, r^{\mathcal{D}}\right) \rho_{\mathrm{C}}\left(x, r^{\mathcal{H}}\right) \mathrm{d} x \\
& +\int_{\Omega} \frac{V^{\mathrm{CC}}}{2 \rho_{0}} \rho_{\mathrm{C}}\left(x, r^{\mathcal{H}}\right) \rho_{\mathrm{C}}\left(x, r^{\mathcal{H}}\right) \mathrm{d} x,
\end{aligned}
$$

where $\mathrm{k}, \mathrm{m} \in\{\mathrm{A}, \mathrm{B}\}$.

At this stage, it is hopeless to compute the free energy and macroscopic densities due to the nonlocal character of the interaction term. As is customary (see [10-14,20,23]), we introduce a mean field approximation whereby the effects of all the chains on a single chain is simulated by means of an external field acting separately on the A-C monomers. In doing so, we avoid these purely technical obstacles of using the delta function.

The mean field approximation is supported (or motivated) by the following variational principle (see, for example, [2]): For any distribution $D^{\prime}$ (i.e. $D^{\prime} \neq D$ ),

$$
\beta \int_{\Gamma} H\left(r^{\mathcal{D}}, r^{\mathcal{H}}\right) D^{\prime}\left(r^{\mathcal{D}}, r^{\mathcal{H}}\right) \mathrm{d} \mu-S\left(D^{\prime}\right)>-\log Z
$$

with equality when $D^{\prime}=D$ on the left side, $D$ being as above the Gibbs canonical distribution induced by (3.3).

Here $S\left(D^{\prime}\right)$ denotes the statistical entropy associated with the distribution $D^{\prime}$, i.e.

$$
S\left(D^{\prime}\right)=-\int D^{\prime} \log D^{\prime} \mathrm{d} \mu .
$$

This variational principle motivates the following approximation method: consider a smaller class of distributions $D^{\prime}$, and define

$$
F\left(D^{\prime}\right)=\int_{\Gamma} H\left(r^{\mathcal{D}}, r^{\mathcal{H}}\right) D^{\prime}\left(r^{\mathcal{D}}, r^{\mathcal{H}}\right) \mathrm{d} \mu-\beta^{-1} S\left(D^{\prime}\right)
$$

$F\left(D^{\prime}\right)$ may be considered as an approximate free energy of the original system under $D^{\prime}$. Assume that in the smaller class $F\left(D^{\prime}\right)$ is easier to compute and minimize. Then the minimizer within this smaller class approximates the true distribution $D$.

In the self-consistent field theory (see Helfand [10], Helfand and Wasserman [11-13], Hong and Noolandi [14], Matsen and Schick [20] - the latter two being formulated in the context of diblock copolymers), we choose the class of distributions to be those generated by triple of external fields $U=\left(U^{\mathrm{A}}, U^{\mathrm{B}}, U^{\mathrm{C}}\right)$, acting on the A-C monomers, 
respectively. There is no interaction between the monomers. These fields induces the following Hamiltonian on $\Gamma$ :

$$
H_{U}\left(r^{\mathcal{D}}, r^{\mathcal{H}}\right)=\sum_{i=1}^{n} \sum_{\mathrm{k}=\mathrm{A}, \mathrm{B}} \int_{\mathcal{I}_{\mathrm{k}}} U^{\mathrm{k}}\left(r_{i}^{\mathcal{D}}(\tau)\right) \mathrm{d} \tau+\sum_{i=1}^{\nu} \int_{0}^{N_{\mathrm{C}}} U^{\mathrm{C}}\left(r_{i}^{\mathcal{H}}(\tau)\right) \mathrm{d} \tau
$$

They in turn induce a Gibbs canonical distribution and partition function:

$$
D_{U}\left(r^{\mathcal{D}}, r^{\mathcal{H}}\right)=\frac{1}{Z_{U}} \exp \left(-\beta H_{U}\left(r^{\mathcal{D}}, r^{\mathcal{H}}\right)\right), \quad Z_{U}=\int_{\Gamma} \exp \left(-\beta H_{U}\left(r^{\mathcal{D}}, r^{\mathcal{H}}\right)\right) \mathrm{d} \mu .
$$

We use $\langle\cdot\rangle_{U}$ to denote the expectation with respect to $D_{U}\left(r^{\mathcal{D}}, r^{\mathcal{H}}\right) \mathrm{d} \mu$. It is straightforward to show (see [6]) that

$$
S\left(D_{U}\right)=\beta \int_{\Omega} \sum_{\mathrm{k}=\mathrm{A}, \mathrm{B}, \mathrm{C}} U^{\mathrm{k}}(x)\left\langle\rho_{\mathrm{k}}(x)\right\rangle_{U}+\log Z_{U} .
$$

The approximate free energy under $U$ is

$$
F(U)=\int_{\Omega}\left[\sum_{\mathrm{k}, \mathrm{m}=\mathrm{A}, \mathrm{B}, \mathrm{C}} \frac{V^{\mathrm{km}}}{2 \rho_{0}}\left\langle\rho_{\mathrm{k}}(x)\right\rangle_{U}\left\langle\rho_{\mathrm{m}}(x)\right\rangle_{U}-\sum_{\mathrm{k}=\mathrm{A}, \mathrm{B}, \mathrm{C}} U^{\mathrm{k}}(x)\left\langle\rho_{\mathrm{k}}(x)\right\rangle_{U}\right] \mathrm{d} x-\frac{1}{\beta} \log Z_{U} .
$$

We note that $Z_{U}$ factors into two parts associated with the diblocks (AB) and homopolymers $(\mathrm{C})$, respectively. That is,

$$
\begin{aligned}
Z_{U}=Z_{\left(U^{\mathrm{A}}, U^{\mathrm{B}}\right)} \cdot Z_{U^{\mathrm{C}}}= & \left\{\int_{\Gamma_{1}^{\mathcal{D}}} \exp \left(-\beta\left[\int_{\mathcal{I}_{\mathrm{A}}} U^{A} \mathrm{~A}\left(r_{1}^{\mathcal{D}}(\tau)\right) \mathrm{d} \tau+\int_{\mathcal{I}_{\mathrm{B}}} U^{\mathrm{B}}\left(r_{1}^{\mathcal{D}}(\tau)\right) \mathrm{d} \tau\right]\right) \mathrm{d} \mu_{1}^{\mathcal{D}}\right\}^{n} \\
& \times\left\{\int_{\Gamma_{1}^{\mathcal{H}}} \exp \left(-\beta \int_{0}^{N_{\mathrm{C}}} U^{\mathrm{C}}\left(r_{1}^{\mathcal{H}}(\tau)\right) \mathrm{d} \tau\right) \mathrm{d} \mu_{1}^{\mathcal{H}}\right\}^{v}
\end{aligned}
$$

Since $\log Z_{U}$, and hence $S_{D_{U}}$ decouple, the free energy can be written as. ${ }^{1}$

$$
\begin{aligned}
F(U)= & F\left(U^{\mathrm{A}}, U^{\mathrm{B}}, U^{\mathrm{C}}\right) \\
= & \int_{\Omega}\left[\sum_{\mathrm{k}, \mathrm{m}=\mathrm{A}, \mathrm{B}, \mathrm{C}} \frac{V^{\mathrm{km}}}{2 \rho_{0}}\left\langle\rho_{\mathrm{k}}(x)\right\rangle_{U}\left\langle\rho_{\mathrm{m}}(x)\right\rangle_{U}-\sum_{\mathrm{k}=\mathrm{A}, \mathrm{B}} U^{\mathrm{k}}(x)\left\langle\rho_{\mathrm{k}}(x)\right\rangle_{\left(U^{\mathrm{A}}, U^{\mathrm{B}}\right)}-U^{\mathrm{C}}(x)\left\langle\rho_{\mathrm{C}}(x)\right\rangle_{U^{\mathrm{C}}}\right] \mathrm{d} x \\
& -\frac{1}{\beta} \log Z_{\left(U^{\mathrm{A}}, U^{\mathrm{B}}\right)}-\frac{1}{\beta} \log Z_{U^{\mathrm{C}}} .
\end{aligned}
$$

Here, we have used the fact that the $\left\{r_{i}^{\mathcal{D}}, r_{j}^{\mathcal{H}}\right\}$ are all independent Brownian chains.

\footnotetext{
${ }^{1}$ We note here, as we should have in [6], that in calculating the expectation of the original interaction Hamiltonian $H\left(r^{\mathcal{D}}, r^{\mathcal{H}}\right)$ under the external field $U$, i.e.,

$$
\int_{\Gamma} H\left(r^{\mathcal{D}}, r^{\mathcal{H}}\right) D_{U}\left(r^{\mathcal{D}}, r^{\mathcal{H}}\right) \mathrm{d} \mu=\int_{\Omega} \sum_{\mathrm{k}, \mathrm{m}=\mathrm{A}, \mathrm{B}, \mathrm{C}} \frac{V^{\mathrm{km}}}{2 \rho_{0}}\left\langle\rho_{\mathrm{k}}(x)\right\rangle_{U}\left\langle\rho_{\mathrm{m}}(x)\right\rangle_{U} \mathrm{~d} x,
$$

we assume independence of the microscopic densities $\rho_{\mathrm{k}}\left(x, r^{\mathcal{D}}\right), \mathrm{k}=\mathrm{A}, \mathrm{B}, \rho_{\mathrm{C}}\left(x, r^{\mathcal{H}}\right)$. The densities involve sums over all the respective chains, and when taking products the diagonal terms (i.e. products involving the same chain) are of course dependent. However, in the end we consider a system of many chains (large $n$ and $v$ ). Hence for any given chain, its energetic interaction with the other chains is of greater order than the interaction with itself. We thus neglect the contribution of the correction term associated with this chain self-interaction.
} 
In this paper, our goal is to write the energy entirely in terms of the macroscopic densities $\left\langle\rho_{\mathrm{k}}(x)\right\rangle_{U}$. In [6], we followed the following steps:

(i) We determine the dependence of $Z_{U}$ and $\left\langle\rho_{\mathrm{k}}(x)\right\rangle_{U}$ on $U$ via the Feynman-Kac integration theory wherein we solve the modified heat equations with Dirichlet boundary conditions.

(ii) We use the self-consistency of the model to write the entropic part of the energy as an inner product of $\left\langle\rho_{\mathrm{k}}(x)\right\rangle_{U}$ and $U$,

(iii) We take the thermodynamic limit to enable us to neglect boundary effects and work with the fundamental solution of the heat equation. We then compute the linearized (about $\beta=0)^{2}$ dependence of $\left\langle\rho_{\mathrm{k}}(x)\right\rangle_{U}$ on $U$.

(iv) In Fourier space, we invert this relationship to obtain $U$ as a function of $\left\langle\rho_{\mathrm{k}}(x)\right\rangle_{U}$, the density distribution it generates.

(v) We return from the thermodynamic limit, to consider a finite system over a finite domain. Lastly, we nondimensionalize.

We carry out essentially the same program separately for the diblocks and the homopolymers. However, we first make one simplification. We take $\Omega=(0, L)^{3}$ with the periodic boundary condition, i.e. the boundaries are properly identified so that $\Omega$ is topologically a three dimensional torus. Thus, the configurations of the chains may be regarded as Brownian motion on a 3D flat torus, and the modified heat equations in the Feynman-Kac theory are solved with periodic boundary conditions. This eliminates the need to take the thermodynamic limit. The modified steps are as follows:

(i') We determine the dependence of $Z_{U}$ and $\left\langle\rho_{\mathrm{k}}(x)\right\rangle_{U}$ on $U$ via the Feynman-Kac integration theory wherein we solve the modified heat equations with periodic boundary conditions.

(ii') We use the self-consistency of the model to write the entropic part of the energy as an inner product of $\left\langle\rho_{\mathrm{k}}(x)\right\rangle_{U}$ and $U$,

(iii') We compute the linearized (about $\beta=0$ ) dependence of $\left\langle\rho_{\mathrm{k}}(x)\right\rangle_{U}$ on $U$.

(iv') In Fourier space, we invert this relationship to obtain $U$ as a function of $\left\langle\rho_{\mathrm{k}}(x)\right\rangle_{U}$, the density distribution it generates.

(v') We nondimensionalize.

For the diblocks, the calculations are verbatim from [6] except for the simplification due to the periodic boundary conditions. One obtains

$$
Z_{\left(U^{\mathrm{A}}, U^{\mathrm{B}}\right)}=\left\{\int_{\Omega} q_{\left(U^{\mathrm{A}}, U^{\mathrm{B}}\right)}(y, 0) \mathrm{d} y\right\}^{n}=\left\{\int_{\Omega} q_{\left(U^{\mathrm{A}}, U^{\mathrm{B}}\right)}^{*}(y, N) \mathrm{d} y\right\}^{n} .
$$

and

$$
\left\langle\rho_{\mathrm{k}}(x)\right\rangle_{\left(U^{\mathrm{A}}, U^{\mathrm{B}}\right)}=\frac{n}{Z_{\left(U^{\mathrm{A}}, U^{\mathrm{B}}\right)}^{1 / n}} \int_{\mathcal{I}_{\mathrm{k}}} q_{\left(U^{\mathrm{A}}, U^{\mathrm{B}}\right)}(x, \tau) q_{\left(U^{\mathrm{A}}, U^{\mathrm{B}}\right)}^{*}(x, \tau) \mathrm{d} \tau .
$$

where $\mathrm{k}=\mathrm{A}, \mathrm{B}$. Where setting $\bar{U}(y, \tau)=U^{\mathrm{k}}(y)$ for $\tau \in \mathcal{I}_{\mathrm{k}}, q_{\left(U^{\mathrm{A}}, U^{\mathrm{B}}\right)}(x, \tau)$ and $q_{\left(U^{\mathrm{A}}, U^{\mathrm{B}}\right)}^{*}(x, \tau)$ solve

$$
\left(q_{\left(U^{\mathrm{A}}, U^{\mathrm{B}}\right)}\right)_{\tau}+\left(l^{2} / 6\right) \Delta q_{\left(U^{\mathrm{A}}, U^{\mathrm{B}}\right)}-\beta \bar{U} q_{\left(U^{\mathrm{A}}, U^{\mathrm{B}}\right)}=0, \quad q_{\left(U^{\mathrm{A}}, U^{\mathrm{B}}\right)}(y, N)=1,(y, \tau) \in \Omega \times(0, N)
$$

\footnotetext{
${ }^{2} \beta=0$ corresponds to infinite temperature, and we refer the reader to [6] for warnings about the validity of such an expansion in the different regimes.
} 
and

$$
\left(q_{\left(U^{\mathrm{A}}, U^{\mathrm{B}}\right)}^{*}\right)_{\tau}-\left(l^{2} / 6\right) \Delta q_{\left(U^{\mathrm{A}}, U^{\mathrm{B}}\right)}^{*}+\beta \bar{U} q_{\left(U^{\mathrm{A}}, U^{\mathrm{B}}\right)}^{*}=0, \quad q_{\left(U^{\mathrm{A}}, U^{\mathrm{B}}\right)}^{*}(y, 0)=1,(y, \tau) \in \Omega \times(0, N),
$$

respectively. Here we invoke periodic boundary conditions on $\partial \Omega \times(0, N)$.

To write $\beta\left(U^{\mathrm{A}}, U^{\mathrm{B}}\right)$ as a function of $\left\langle\rho_{\mathrm{k}}(x)\right\rangle_{\left(U^{\mathrm{A}}, U^{\mathrm{B}}\right)}, \mathrm{k}=\mathrm{A}, \mathrm{B}$ we linearize about $\beta=0$. We find ${ }^{3}$

$$
\begin{aligned}
E_{\text {entropy }}^{\mathcal{D}} & :=-S\left(D_{\left(U^{\mathrm{A}}, U^{\mathrm{B}}\right)}^{\mathcal{D}}\right)+S\left(D_{0}^{\mathcal{D}}\right) \\
& \approx \frac{1}{2 \rho_{0}^{\mathcal{D}}} \int_{\Omega}\left[\sum_{\mathrm{k} \in\{\mathrm{A}, \mathrm{B}\}} \frac{l^{2} M^{\mathrm{kk}}}{6}\left|\nabla \rho_{\mathrm{k}}\right|^{2}+\sum_{\mathrm{k}, \mathrm{m} \in\{\mathrm{A}, \mathrm{B}\}} \frac{6 R^{\mathrm{km}}}{l^{2} N^{2}}\left((-\Delta)^{-1}\left(\rho_{\mathrm{k}}-\bar{\rho}_{\mathrm{k}}\right)\right)\left(\rho_{\mathrm{m}}-\bar{\rho}_{\mathrm{m}}\right)\right] \mathrm{d} x,
\end{aligned}
$$

where

$$
M=\frac{1}{2}\left[\begin{array}{cc}
\frac{N}{N_{\mathrm{A}}} & 0 \\
0 & \frac{N}{N_{\mathrm{B}}}
\end{array}\right], \quad R=\frac{3}{2}\left[\begin{array}{cc}
\frac{N^{2}}{N_{\mathrm{A}}^{2}} & -\frac{N^{2}}{N_{\mathrm{A}} N_{\mathrm{B}}} \\
-\frac{N^{2}}{N_{\mathrm{A}} N_{\mathrm{B}}} & \frac{N^{2}}{N_{\mathrm{B}}^{2}}
\end{array}\right],
$$

and $\rho_{0}^{\mathcal{D}}$ is defined by (3.2). In (3.12) above, we have for convenience denoted $\left\langle\rho_{\mathrm{k}}(x)\right\rangle_{\left(U^{\mathrm{A}}, U^{\mathrm{B}}\right)}, \mathrm{k}=\mathrm{A}, \mathrm{B}$ and $\left\langle\rho_{\mathrm{C}}(x)\right\rangle_{U^{\mathrm{C}}}$ simply by $\rho_{\mathrm{k}}(x), \mathrm{k}=\mathrm{A}, \mathrm{B}$ and $\rho_{\mathrm{C}}(x)$, respectively.

For the homopolymers, Steps (i') and (ii') are the same; That is,

$$
Z_{U^{\mathrm{C}}}=\left\{\int_{\Omega} q_{U^{\mathrm{C}}}(y, 0) \mathrm{d} y\right\}^{v}=\left\{\int_{\Omega} q_{U^{\mathrm{C}}}^{*}\left(y, N_{\mathrm{C}}\right) \mathrm{d} y\right\}^{\nu},
$$

and

$$
\left\langle\rho_{\mathrm{C}}(x)\right\rangle_{U^{\mathrm{C}}}=\frac{v}{Z_{U^{\mathrm{C}}}^{1 / v}} \int_{0}^{N_{\mathrm{C}}} q_{U^{\mathrm{C}}}(x, \tau) q_{U^{\mathrm{C}}}^{*}(x, \tau) \mathrm{d} \tau,
$$

where $q_{U^{\mathrm{C}}}$ and $q_{U^{\mathrm{C}}}^{*}$ solve (3.9) and (3.10), respectively, with $\bar{U}$ replaced with $U^{\mathrm{C}}$. Step (iii') is however different, and indeed simpler since only one field acts on the homopolymers. We will use the linear approximation:

$$
\left\langle\rho_{\mathrm{C}}\right\rangle_{U^{\mathrm{C}}} \approx \bar{\rho}_{\mathrm{C}}-\frac{\rho_{0}^{\mathcal{H}}}{N_{\mathrm{C}}} R *\left(\beta U^{\mathrm{C}}\right) .
$$

Using the Fourier series of the fundamental solution to the heat equation with periodic boundary conditions

$$
\mathcal{K}(z, t)=\sum_{\xi \in Z^{3}} \frac{1}{L^{3}} \exp \left(-\frac{l^{2}}{6}\left(\frac{2 \pi}{L}\right)^{2} \xi^{2}|t|\right) \exp \left(\frac{2 \pi i}{L} \xi \cdot z\right) .
$$

one obtains the Fourier series of $R$ :

$$
R(z)=\sum_{\xi \in Z^{3}} \frac{2}{L^{3}}\left|\frac{2 \pi l \xi}{\sqrt{6} L}\right|^{-4} h\left(\frac{4 \pi^{2} l^{2}|\xi|^{2} N_{\mathrm{C}}}{6 L^{2}}\right) \exp \left(\frac{2 \pi i}{L} \xi \cdot z\right) .
$$

\footnotetext{
${ }^{3}$ This follows directly from [6] with the following simplifications: the periodic boundary conditions imply that the solutions $q_{0}$ and $q_{0}^{*}$ (i.e. under a external field $\left(U^{\mathrm{A}}, U^{\mathrm{B}}\right)=0$ ) are identically equal to 1 , and the solution $Q_{0}$ (see [6]) is simply the fundamental solution of the heat equation with periodic boundary conditions.
} 
where

$$
h(s)=e^{-s}+s-1 .
$$

Denote the Fourier coefficients of $R$ by

$$
\hat{R}(\xi)=\frac{2}{L^{3}}\left|\frac{2 \pi l \xi}{\sqrt{6} L}\right|^{-4} h\left(\frac{4 \pi^{2} l^{2}|\xi|^{2} N_{\mathrm{C}}}{6 L^{2}}\right)
$$

so that

$$
R(z)=\sum_{\xi \in \mathbf{Z}^{3}} \hat{R}(\xi) \exp \left(\frac{2 \pi i}{L} \xi \cdot z\right)
$$

For Step (iv'), we compute the inverse of $\hat{R}$ via the following approximation:

$$
\begin{array}{ll}
h(s) \approx s & \text { if } s \gg 1 \\
h(s) \approx \frac{s^{2}}{2} & \text { if } s \ll 1 .
\end{array}
$$

This leads to

$$
\frac{1}{\hat{R}(\xi)} \approx \frac{(2 \pi l|\xi|)^{2} L}{12 N_{\mathrm{C}}}+\frac{L^{3}}{N_{\mathrm{C}}^{2}}
$$

Hence, denoting $\left\langle\rho_{k}\right\rangle_{U^{\mathrm{C}}}$ simply by $\rho_{\mathrm{C}}$, we obtain

$$
\beta U^{\mathrm{C}} \approx-\frac{N_{\mathrm{C}}}{\rho_{0}^{\mathcal{H}}} T\left(\rho_{\mathrm{C}}-\bar{\rho}_{\mathrm{C}}\right)
$$

where $T$ is the operator

$$
T:=\frac{l^{2}}{12 N_{\mathrm{C}}}(-\triangle)+\frac{1}{N_{\mathrm{C}}^{2}}
$$

and $\rho_{0}^{\mathcal{H}}$ is defined by (3.2). Integrating with respect to $\left(\rho_{\mathrm{C}}-\bar{\rho}_{\mathrm{C}}\right)$, we find

$$
E_{\text {entropy }}^{\mathcal{H}}:=-S\left(D_{U^{\mathrm{C}}}^{\mathcal{H}}\right)+S\left(D_{0}^{\mathcal{H}}\right) \approx \frac{1}{2 \rho_{0}^{\mathcal{H}}} \int_{\Omega}\left[\frac{l^{2}}{12}\left|\nabla \rho_{\mathrm{C}}\right|^{2}+\frac{1}{N_{\mathrm{C}}}\left(\rho_{\mathrm{C}}-\bar{\rho}_{\mathrm{C}}\right)^{2}\right] \mathrm{d} x .
$$

For both the diblocks and homopolymers we neglect the constants $S\left(D_{0}^{\mathcal{D}}\right), S\left(D_{0}^{\mathcal{H}}\right)$.

From (3.11), (3.20), the free energy (3.6) becomes 


$$
\begin{aligned}
\beta F= & \int_{\Omega}\left[\frac{1}{2 \rho_{0}^{\mathcal{D}}} \sum_{\mathrm{k} \in\{\mathrm{A}, \mathrm{B}\}} \frac{l^{2} M^{\mathrm{kk}}}{6}\left|\nabla \rho_{\mathrm{k}}\right|^{2}+\frac{1}{2 \rho_{0}^{\mathcal{H}}} \frac{l^{2}}{12}\left|\nabla \rho_{\mathrm{C}}\right|^{2}\right. \\
& +\sum_{\mathrm{k}, \mathrm{m} \in\{\mathrm{A}, \mathrm{B}\}} \frac{6 L^{\mathrm{km}}}{2 \rho_{0}^{\mathcal{D}} l^{2} N^{2}}\left((-\Delta)^{-1}\left(\rho_{\mathrm{k}}-\bar{\rho}_{\mathrm{k}}\right)\right)\left(\rho_{\mathrm{m}}-\bar{\rho}_{\mathrm{m}}\right) \\
& \left.+\frac{1}{2 \rho_{0}^{\mathcal{H}} N_{\mathrm{C}}}\left(\rho_{\mathrm{C}}-\bar{\rho}_{\mathrm{C}}\right)^{2}+\sum_{\mathrm{k}, \mathrm{m}=\mathrm{A}, \mathrm{B}, \mathrm{C}} \frac{\beta V^{\mathrm{km}}}{2 \rho_{0}} \rho_{\mathrm{k}} \rho_{\mathrm{m}}\right] \mathrm{d} x,
\end{aligned}
$$

where $F=F\left(\rho_{\mathrm{A}}, \rho_{\mathrm{B}}, \rho_{\mathrm{C}}\right)$ is the total free energy.

We scale the variable $x$ to $L x$ so that $x \in(0,1)^{3}$. We also re-scale $\rho_{\mathrm{k}}$ to $u_{\mathrm{k}}=\rho_{\mathrm{k}} / \rho_{0}$. Then the total free energy per unit monomer in dimensionless units is 4

$$
E(u):=\frac{\beta F}{\rho_{0} L^{3}}=\int_{T^{3}}\left[\frac{\varepsilon^{2}}{2}\langle K \nabla u, \nabla u\rangle+\frac{\varepsilon \gamma}{2}\left\langle J(-\Delta)^{-1 / 2}(u-\bar{u}),(-\Delta)^{-1 / 2}(u-\bar{u})\right\rangle+W(u)\right] \mathrm{d} x
$$

where

$$
\begin{aligned}
& \varepsilon^{2}=\frac{l^{2}}{6 L^{2}} \quad \text { and } \quad \varepsilon \gamma=\frac{6 L^{2}}{l^{2} N^{2}}\left(\frac{n N}{n N+v N_{\mathrm{C}}}\right), \\
& K=\frac{1}{2}\left[\begin{array}{cccc}
\frac{n N+v N_{\mathrm{C}}}{n N_{\mathrm{A}}} & 0 & 0 & \\
0 & \frac{n N+v N_{\mathrm{C}}}{n N_{\mathrm{B}}} & 0 \\
0 & 0 & \frac{n N+v N_{\mathrm{C}}}{v N_{\mathrm{C}}}
\end{array}\right], \\
& J=\frac{3}{2}\left[\begin{array}{ccc}
\left(\frac{n N+v N_{\mathrm{C}}}{n N_{\mathrm{A}}}\right)^{2} & -\frac{\left(n N+v N_{\mathrm{C}}\right)^{2}}{n N_{\mathrm{A}} n N_{\mathrm{B}}} & 0 \\
-\frac{\left(n N+v N_{\mathrm{C}}\right)^{2}}{n N_{\mathrm{A}} n N_{\mathrm{B}}} & \left(\frac{n N+v N_{\mathrm{C}}}{n N_{\mathrm{B}}}\right)^{2} & 0 \\
0 & 0 & 0
\end{array}\right], \\
& W(u)=\sum_{\mathrm{k}, \mathrm{m} \in\{\mathrm{A}, \mathrm{B}, \mathrm{C}\}} \frac{\beta V^{\mathrm{km}} u_{\mathrm{k}} u_{\mathrm{m}}}{2} .
\end{aligned}
$$

We have denoted the three-dimensional flat torus of the unit length to be $T^{3}$. Note that in the calculation of (3.22), we have ignored the second to last term of (3.21). We can justify this in two separate ways. One expects minimizers $\left(u_{\mathrm{A}}, u_{\mathrm{B}}, u_{\mathrm{C}}\right)$ to take on primarily the values $(1,0,0),(0,1,0)$ and $(0,0,1)$. Thus, this term involving the integral of $\left(u-u_{\mathrm{C}}\right)^{2}$ may be regarded as a constant. Alternatively, since $N_{\mathrm{C}}$ is large, one may ignore this term because it is much smaller than the last term of (3.21).

\footnotetext{
${ }^{4}$ The reader may be surprised as to the dependence on $N_{\mathrm{C}}$ in all the non-zero components of $K$, and $J$. This is simpy because of the rescaling of variables whereby all the monomer densities are scaled via the total monomer density $\rho_{0}$.
} 
We set

$$
a=\frac{n N_{\mathrm{A}}}{n N+v N_{\mathrm{C}}}, \quad b=\frac{n N_{\mathrm{B}}}{n N+v N_{\mathrm{C}}}, \quad c=\frac{v N_{\mathrm{C}}}{n N+v N_{\mathrm{C}}} .
$$

Then $a+b+c=1$ and

$$
K=\frac{1}{2}\left[\begin{array}{ccc}
\frac{1}{a} & 0 & 0 \\
0 & \frac{1}{b} & 0 \\
0 & 0 & \frac{1}{c}
\end{array}\right], \quad J=\frac{3}{2}\left[\begin{array}{ccc}
\frac{1}{a^{2}} & -\frac{1}{a b} & 0 \\
-\frac{1}{a b} & \frac{1}{b^{2}} & 0 \\
0 & 0 & 0
\end{array}\right] .
$$

We assume that $W(u)$ is concave in $u_{\mathrm{A}}+u_{\mathrm{B}}+u_{\mathrm{C}}=1$. This is achieved if $V^{\mathrm{km}}$ is large compared to $V^{\mathrm{kk}}$ and $V^{\mathrm{mm}}$ for all $\mathrm{k}, \mathrm{m} \in\{\mathrm{A}, \mathrm{B}, \mathrm{C}\}, \mathrm{k} \neq \mathrm{m}$. We subtract a linear term

$$
\sum_{\mathrm{k} \in\{\mathrm{A}, \mathrm{B}, \mathrm{C}\}} \frac{\beta V^{\mathrm{kk}}}{2} u_{\mathrm{k}}
$$

from $W$ and redefine $W$ as follows:

$$
W(u)=\sum_{\mathrm{k}, \mathrm{m} \in\{\mathrm{A}, \mathrm{B}, \mathrm{C}\}} \frac{\beta V^{\mathrm{km}} u_{\mathrm{k}} u_{\mathrm{m}}}{2}-\sum_{\mathrm{k} \in\{\mathrm{A}, \mathrm{B}, \mathrm{C}\}} \frac{\beta V^{\mathrm{kk}}}{2} u_{\mathrm{k}} .
$$

This $W$ is non-negative, and it achieves minimum value 0 at exactly three points: $(1,0,0),(0,1,0)$, and $(0,0,1)$. Note that this modification of $W$ only changes the free energy by a constant.

\section{Local minimizers in 1D: the CABAB ... ABA lamellar pattern}

In this last section, we prove a result (Theorem 4.4) about local minimizers of (3.22) within the ansatz of patterns depending only on one variable. In one space dimension one naturally conjectures that the optimal pattern must consist of two macrodomains; one involving the pure $\mathrm{C}$ phase and the other devoted to a lamellar structure of alternating $\mathrm{A}$ and $\mathrm{B}$ microdomains. The respective widths of these domains would be dictated by the material parameters including $a, b$ and $c$. We call such a pattern the CABAB ... ABA lamellar pattern. While we are not able prove this structure is the global minimizer, we prove the existence of local minimizers displaying this pattern. Owing to the fact that such a structure has only one $\mathrm{C}$ domain but many $\mathrm{A}$ and $\mathrm{B}$ domains, let us agree to refer to the pure $\mathrm{A}$ and $\mathrm{B}$ domains as microdomains and the pure $\mathrm{C}$ domain as a macrodomain.

Let $u=\left(u_{\mathrm{A}}, u_{\mathrm{B}}, u_{\mathrm{C}}\right)$ be the relative monomer density fields. In one space dimension, the free energy (3.22) becomes

$$
I_{\varepsilon}(u):=\int_{0}^{1}\left[\frac{\varepsilon^{2}}{2}\left\langle K u^{\prime}, u^{\prime}\right\rangle+\frac{\varepsilon \gamma}{2}\left\langle J(-\Delta)^{-1 / 2}(u-\bar{u}),(-\Delta)^{-1 / 2}(u-\bar{u})\right\rangle+W(u)\right] \mathrm{d} x
$$

where $K$ and $J$ are defined by (3.27) and $W$ is defined for $u$ that satisfies $u_{\mathrm{A}}+u_{\mathrm{B}}+u_{\mathrm{C}}=1$. $W$ achieves minimum value 0 at exactly three points: $(1,0,0),(0,1,0)$ and $(0,0,1)$. The functional $I_{\varepsilon}$ is defined in

$$
\left\{u=\left(u_{\mathrm{A}}, u_{\mathrm{B}}, u_{\mathrm{C}}\right): u_{\mathrm{m}} \in W^{1,2}\left(T^{1}\right)(\mathrm{m}=\mathrm{A}, \mathrm{B}, \mathrm{C}), u_{\mathrm{A}}+u_{\mathrm{B}}+u_{\mathrm{C}}=1, \bar{u}_{\mathrm{A}}-a=\bar{u}_{\mathrm{B}}-b=\bar{u}_{\mathrm{C}}-c=0\right\} .
$$

Here we use $T^{1}$ to denote $(0,1)$ with 0 and 1 identified. 
For $\varepsilon$ small, the theory of $\Gamma$-convergence (cf. $[8,4,1]$ ) allows us to reduce the study of $I_{\varepsilon}$ to that of a much simpler problem, which we call $Q$ (the $\Gamma$-limit of $\varepsilon^{-1} I_{\varepsilon}$ ) and define below in (4.34). We will give a precise statement of this convergence in Lemma 4.5. The functional $Q$ will be defined on the admissible set

$$
\left\{u=\left(u_{\mathrm{A}}, u_{\mathrm{B}}, u_{\mathrm{C}}\right): u_{\mathrm{A}}+u_{\mathrm{B}}+u_{\mathrm{C}}=1, \bar{u}_{\mathrm{A}}-a=\bar{u}_{\mathrm{B}}-b=\bar{u}_{\mathrm{C}}-c=0, u_{\mathrm{A}}, u_{\mathrm{B}}, u_{\mathrm{C}} \in B V\left(T^{1},\{0,1\}\right)\right\} .
$$

Here $B V\left(T^{1},\{0,1\}\right)$ is the space of functions of bounded variation that only take two values: 0 and 1 . Each member in this function space jumps between 0 and 1 at finitely many points. Therefore each $u$ from (4.32) divides $T^{1}$ into a number of intervals where $u_{\mathrm{A}}=1$ and $u_{\mathrm{B}}=u_{\mathrm{C}}=0$ (pure A domains), a number of intervals where $u_{\mathrm{B}}=1$ and $u_{\mathrm{A}}=u_{\mathrm{C}}=0$ (pure $\mathrm{B}$ domains), and a number of intervals where $u_{\mathrm{C}}=1$ and $u_{\mathrm{A}}=u_{\mathrm{B}}=0$ (pure $\mathrm{C}$ domains).

The set (4.32) is much simpler than the set (4.31), because of a natural decomposition. A pattern $\mathrm{p}$ of $\mathrm{k}$ microdomains is a loop of $\mathrm{k}$ letters of A-C modulo translation. Note that unlike in the previous section, $\mathrm{k}$ will now denote a positive integer. Any two adjacent letters must be distinct. The first and the last letter are considered adjacent. For example, $p=C A B A B A$ is a pattern of six subdomains. Note that ACABAB is by definition the same pattern because they differ just by a translation. As explained above each member of (4.32) has a particular pattern $\mathrm{p}$, and (4.32) is the disjoint union of the sets

$$
\{u \in(4.32): u \text { has the pattern } \mathrm{p}\}
$$

that are labeled by $p$. Between letters of a pattern we have interfaces. For $p=$ CABABA, there is a CA interface between the first two letters, an $\mathrm{AB}$ interface between the second letter and the third letter, etc. We also have another CA interface between the last letter and the first letter.

We are now ready to define $Q$. For each $u$ in (4.32),

$$
Q(u)=\tau^{\mathrm{AB}} N_{\mathrm{AB}}+\tau^{\mathrm{BC}} N_{\mathrm{BC}}+\tau^{\mathrm{CA}} N_{\mathrm{CA}}+\frac{\gamma}{2} \int_{0}^{1}\left\langle J(-\Delta)^{-1 / 2}(u-\bar{u}),(-\Delta)^{-1 / 2}(u-\bar{u})\right\rangle \mathrm{d} x .
$$

Here $N_{\mathrm{AB}}$ (and $N_{\mathrm{BC}}, N_{\mathrm{CA}}$ ) is the number of $\mathrm{AB}$ (BC and CA, respectively) interfaces. $\tau^{\mathrm{AB}}, \tau^{\mathrm{BC}}$, and $\tau^{\mathrm{CA}}$ are positive constants called surface tensions. For example, $\tau^{\mathrm{AB}}$ is the surface tension of an $\mathrm{AB}$ interface, and is given by (cf. Lemma 4.5 and [1])

$$
\tau^{\mathrm{AB}}=\inf _{\eta}\left\{\sqrt{2} \int_{0}^{1} \sqrt{W(\eta(t))\left\langle K \eta^{\prime}(t), \eta^{\prime}(t)\right\rangle} \mathrm{d} t: \eta \in C^{1}[0,1], \eta(0)=(1,0,0), \eta(1)=(0,1,0)\right\} .
$$

In this definition $\eta$ is a curve in the plane $u_{\mathrm{A}}+u_{\mathrm{B}}+u_{\mathrm{C}}=1$ connecting the A-state and the B-state. The surface tensions $\tau^{\mathrm{BC}}$ and $\tau^{\mathrm{CA}}$ are similarly defined. Here $K$ and $W$ are given respectively by (3.27) and (3.29). One readily checks that the infimum for $\tau^{\mathrm{AB}}$ is obtained when $\eta(t)=(1,0,0)^{\mathrm{T}}(1-t)+(0,1,0)^{\mathrm{T}} t$, and hence

$$
\tau^{\mathrm{AB}}=\left(\int_{0}^{1} \sqrt{(1-t) t} \mathrm{~d} t\right) \sqrt{\left(\frac{1}{a}+\frac{1}{b}\right) \chi^{\mathrm{AB}}}=\frac{\pi}{8} \sqrt{\left(\frac{1}{a}+\frac{1}{b}\right) \chi^{\mathrm{AB}}}
$$

where the Flory-Huggins AB parameter is given by

$$
\chi^{\mathrm{AB}}:=\beta V^{\mathrm{AB}}-\frac{\beta}{2}\left(V^{\mathrm{AA}}+V^{\mathrm{BB}}\right) .
$$


Similarly, one has

$$
\tau^{\mathrm{AC}}=\frac{\pi}{8} \sqrt{\left(\frac{1}{a}+\frac{1}{c}\right) \chi^{\mathrm{AC}}} \quad \text { and } \quad \tau^{\mathrm{BC}}=\frac{\pi}{8} \sqrt{\left(\frac{1}{b}+\frac{1}{c}\right) \chi^{\mathrm{BC}}}
$$

where

$$
\chi^{\mathrm{AC}}:=\beta V^{\mathrm{AC}}-\frac{\beta}{2}\left(V^{\mathrm{AA}}+V^{\mathrm{CC}}\right) \quad \text { and } \quad \chi^{\mathrm{BC}}:=\beta V^{\mathrm{BC}}-\frac{\beta}{2}\left(V^{\mathrm{BB}}+V^{\mathrm{CC}}\right) .
$$

All three Flory-Huggins parameters $\chi^{\mathrm{AB}}, \chi^{\mathrm{AC}}$ and $\chi^{\mathrm{BC}}$ are assumed a priori to be strictly positive.

With these functionals in hand, we now turn toward their local minima. First we note that the $2 \times 2$ matrix

$$
\left[\begin{array}{ll}
J^{\mathrm{AA}} & J^{\mathrm{AB}} \\
J^{\mathrm{AB}} & J^{\mathrm{BB}}
\end{array}\right]=\frac{3}{2}\left[\begin{array}{cc}
a^{-2} & -(a b)^{-1} \\
-(a b)^{-1} & b^{-2}
\end{array}\right]
$$

has an eigenvalue 0 whose associated eigenvector is $(a, b)$ and a positive eigenvalue $1 / a^{2}+1 / b^{2}$ whose associated eigenvector is $(b,-a)$.

Next we note that the nonlocal part of $I_{\varepsilon}$ and $Q$ can be greatly simplified by introducing the variables

$$
\phi:=b u_{\mathrm{A}}-a u_{\mathrm{B}}, \quad \psi:=a u_{\mathrm{A}}+b u_{\mathrm{B}},
$$

together with $w$ and $z$ where

$$
-w^{\prime \prime}=\phi \text { in } T^{1}, \bar{w}=0 ; \quad-z^{\prime \prime}=\psi-\left(a^{2}+b^{2}\right) \text { in } T^{1}, \bar{z}=0 .
$$

Recall that $\bar{w}$ denotes the average of $w$ over $T^{1}$. To this end, if $v=\left(v_{\mathrm{A}}, v_{\mathrm{B}}, v_{\mathrm{C}}\right)$ is given by

$$
-v_{\mathrm{m}}^{\prime \prime}=u_{\mathrm{m}}-\bar{u}_{\mathrm{m}} \text { in } T^{1}, \bar{v}_{\mathrm{m}}=0, \quad \text { for } \mathrm{m}=\mathrm{A}, \mathrm{B}, \mathrm{C} .
$$

then

$$
w=b v_{\mathrm{A}}-a v_{\mathrm{B}}, z=a v_{\mathrm{A}}+b v_{\mathrm{B}} ; \quad v_{\mathrm{A}}=\frac{a z+b w}{a^{2}+b^{2}}, v_{\mathrm{B}}=\frac{b z-a w}{a^{2}+b^{2}} .
$$

The nonlocal part of $I_{\varepsilon}$ and $Q$ can be rewritten as

$$
\begin{aligned}
\int_{0}^{1} & \left\langle J(-\Delta)^{-1 / 2}(u-\bar{u}),(-\Delta)^{-1 / 2}(u-\bar{u})\right\rangle \mathrm{d} x \\
& =\int_{0}^{1}\left\langle J v^{\prime}, v^{\prime}\right\rangle \mathrm{d} x \\
& =\frac{1}{\left(a^{2}+b^{2}\right)^{2}} \int_{0}^{1}\left[J^{\mathrm{AA}}\left(a z^{\prime}+b w^{\prime}\right)^{2}+2 J^{\mathrm{AB}}\left(a z^{\prime}+b w^{\prime}\right)\left(b z^{\prime}-a w^{\prime}\right)+J^{\mathrm{BB}}\left(b z^{\prime}-a w^{\prime}\right)^{2}\right] \mathrm{d} x \\
& =\frac{1}{\left(a^{2}+b^{2}\right)^{2}} \int_{0}^{1}\left(b^{2} J^{\mathrm{AA}}-2 a b J^{\mathrm{AB}}+a^{2} J^{\mathrm{BB}}\right)\left(w^{\prime}\right)^{2} \mathrm{~d} x=\frac{3}{2 a^{2} b^{2}} \int_{0}^{1}\left(w^{\prime}\right)^{2} \mathrm{~d} x .
\end{aligned}
$$

Hence in what follows, we focus on

$$
\tilde{Q}(u)=\int_{0}^{1}\left(w^{\prime}\right)^{2} \mathrm{~d} x
$$


We now consider a particular pattern $\mathrm{CABAB} \ldots \mathrm{ABA}$ and show that a function $u$ of such a pattern exists as a local minimizer of $Q$. We let $x_{1}, x_{2}, \ldots, x_{k} \in(0,1)$ be the interfaces. Collectively we set $\mathbf{x}=\left(x_{1}, x_{2}, \ldots, x_{k}\right)$. The intervals $\left(0, x_{1}\right)$ and $\left(x_{k}, 1\right)$ are occupied by $\mathrm{C}$-monomers. Since under the periodic boundary condition, 0 and 1 are identified, we should regard $\left(0, x_{1}\right) \cup\left(x_{k}, 1\right)$ as a single interval of $\mathrm{C}$-monomers. The A-monomers occupy the intervals $\left(x_{1}, x_{2}\right),\left(x_{3}, x_{4}\right), \ldots,\left(x_{k-1}, x_{k}\right)$; and the B-monomers occupy the intervals $\left(x_{2}, x_{3}\right),\left(x_{4}, x_{5}\right), \ldots,\left(x_{k-2}, x_{k-1}\right)$. Note that $k$ is even and there is one C-macrodomain, $k / 2 \mathrm{~A}$-microdomains, and $(k / 2)-1 \mathrm{~B}$-microdomains. We view $\tilde{Q}$ as a function of $\mathbf{x}$. Since the function $\phi$ is

$$
\phi(x)=\left\{\begin{array}{lll}
0 & \text { if } & x \in\left(0, x_{1}\right) \\
b & \text { if } & x \in\left(x_{1}, x_{2}\right) \\
-a & \text { if } & x \in\left(x_{2}, x_{3}\right) \\
\vdots & & \\
-a & \text { if } & x \in\left(x_{k-2}, x_{k-1}\right) \\
b & \text { if } & x \in\left(x_{k-1}, x_{k}\right) \\
0 & \text { if } & x \in\left(x_{k}, 1\right)
\end{array},\right.
$$

we sometimes write $\phi(x ; \mathbf{x})$ to emphasize that $\phi$ depends on $\boldsymbol{x}$. Similarly we may write $w(x ; \boldsymbol{x})$.

There exist constraints on $\mathbf{x}$. In particular, $\bar{\phi}=0$ and $\bar{u}_{\mathrm{C}}=c$ imply respectively that

$$
b\left(x_{2}-x_{1}\right)-a\left(x_{3}-x_{2}\right)+\cdots+b\left(x_{k}-x_{k-1}\right)=0, \quad x_{k}-x_{1}=1-c .
$$

Lemma 4.1. If $\mathbf{x}=\left(x_{1}, x_{2}, \ldots, x_{k}\right)$ is a critical point of $\tilde{Q}$ under the constraints (4.44), then

$$
\begin{aligned}
& 2\left(x_{2}-x_{1}\right)=x_{4}-x_{3}=\cdots=x_{k-2}-x_{k-3}=2\left(x_{k}-x_{k-1}\right)=\frac{2 a}{k-2}, \\
& x_{3}-x_{2}=x_{5}-x_{4}=\cdots=x_{k-1}-x_{k-2}=\frac{2 b}{k-2} .
\end{aligned}
$$

Proof. We calculate the derivatives of $\tilde{Q}$.

$$
\frac{\partial \tilde{Q}}{\partial x_{2}}=\frac{\partial}{\partial x_{2}} \int_{0}^{1} w \phi \mathrm{d} x=\frac{\partial}{\partial x_{2}}\left[b \int_{x_{1}}^{x_{2}} w \mathrm{~d} x-a \int_{x_{2}}^{x_{3}} w \mathrm{~d} x+\cdots\right]=(a+b) w\left(x_{2} ; \mathbf{x}\right)+\int_{0}^{1} \phi(x ; \mathbf{x}) \frac{\partial w(x ; \mathbf{x})}{\partial x_{2}} \mathrm{~d} x .
$$

Denoting the Green function of $-\Delta$ by $G$ we find that

$$
\begin{aligned}
\frac{\partial w(x ; \mathbf{x})}{\partial x_{2}} & =\frac{\partial}{\partial x_{2}} \int_{0}^{1} G(x-y) \phi(y ; \mathbf{x}) \mathrm{d} y=\frac{\partial}{\partial x_{2}}\left[b \int_{x_{1}}^{x_{2}} G(x-y) \mathrm{d} y-a \int_{x_{2}}^{x_{3}} G(x-y)+\mathrm{d} y \cdots\right] \\
& =(a+b) G\left(x-x_{2}\right) .
\end{aligned}
$$

Now we return to find

$$
\frac{\partial \tilde{Q}}{\partial x_{2}}=(a+b) w\left(x_{2} ; \mathbf{x}\right)+(a+b) \int_{0}^{1} G\left(x-x_{2}\right) \phi(x ; \mathbf{x}) \mathrm{d} x=2(a+b) w\left(x_{2} ; \mathbf{x}\right) .
$$

Similar calculations applied to $x_{3}, x_{4}, \ldots, x_{k-1}$ yield that

$$
\frac{\partial \tilde{Q}}{\partial x_{j}}=2(-1)^{j}(a+b) w\left(x_{j} ; \mathbf{x}\right), \quad j=2,3, \ldots, k-1 .
$$


Small differences appear when $\tilde{Q}$ is differentiated with respect to $x_{1}$ and $x_{k}$. We find that

$$
\frac{\partial \tilde{Q}}{\partial x_{1}}=-2 b w\left(x_{1} ; \mathbf{x}\right), \quad \frac{\partial \tilde{Q}}{\partial x_{k}}=2 b w\left(x_{k} ; \mathbf{x}\right) .
$$

Every critical point $\mathbf{x}$ of $\tilde{Q}$, or of $Q$, must satisfy the equations

$$
\begin{aligned}
& 2(a+b) w\left(x_{j} ; \mathbf{x}\right)+(a+b) \lambda=0 \quad(j=2,3, \ldots, k-1), \\
& 2 b w\left(x_{1} ; \mathbf{x}\right)+b \lambda+\mu=0, \quad \text { and } \quad 2 b w\left(x_{k} ; \mathbf{x}\right)+b \lambda+\mu=0
\end{aligned}
$$

where $\lambda$ and $\mu$ are the Lagrange multipliers from the constraints (4.44).

Since $w$ solves $-w^{\prime \prime}=\phi$ and $w \in C^{1}[0,1]$, the fact that $w\left(x_{j} ; \mathbf{x}\right)$ is the same for $j=2,3, \ldots, k-1$ implies that all the B-microdomains are of the same length and all the A-microdomains, excluding the first one and the last one, are also of the same length. Also $w\left(x_{1} ; \mathbf{x}\right)=w\left(x_{k} ; \mathbf{x}\right)$ and $w^{\prime \prime}=0$ on the C-macrodomain imply that $w^{\prime}(x ; \mathbf{x})=0$ on the $\mathrm{C}$-macrodomain and particularly $w^{\prime}\left(x_{1} ; \mathbf{x}\right)=w^{\prime}\left(x_{k} ; \mathbf{x}\right)=0$. The length of the first and the last A-microdomains is exactly half of the length of the other A-microdomains. Therefore, the length of each A-microdomain, excluding the first and the last, is $2 a /(k-2)$; and the length of each B-microdomain is $2 b /(k-2)$.

We remark why the pattern we consider is $\mathrm{CABAB} \ldots \mathrm{ABA}$, not CABAB ... AB. If instead we study the $\mathrm{CABAB} \ldots \mathrm{AB}$ pattern, then $k$ is odd and the second equation in (4.48) becomes

$$
\frac{\partial \tilde{Q}}{\partial x_{k}}=-2 a w\left(x_{k} ; \mathbf{x}\right) \text {. }
$$

Also we have $-a$ in front of $\left(x_{k}-x_{k-1}\right)$ in (4.44) and the last equation in (4.49) is changed to

$$
-2 a w\left(x_{k} ; \mathbf{x}\right)-a \lambda+\mu=0 .
$$

Then $w\left(x_{1} ; \mathbf{x}\right) \neq w\left(x_{k} ; \mathbf{x}\right)$ and $w^{\prime} \not \equiv 0$ on $\left(0, x_{1}\right) \cup\left(x_{k}, 1\right)$. We can still prove that

$$
x_{3}-x_{2}=x_{5}-x_{4}=\cdots=x_{k-2}-x_{k-3}, \quad x_{4}-x_{3}=x_{6}-x_{5}=\cdots=x_{k-1}-x_{k-2} .
$$

However, $x_{2}-x_{1}$ is not half of the second quantity in (4.52) and $x_{k}-x_{k-1}$ is not half of the first quantity in (4.52). The exact values of (4.52), $x_{2}-x_{1}$ and $x_{k}-x_{k-1}$ may be determined from $a, b$ and $k$ in a way more complex than $(4.45,4.46)$.

We use an indirect argument to show that the solution $(4.45,4.46)$ is a strict local minimizer, modulo translation. For $\mathbf{x}$ associated with any $u$ in the admissible set (4.32), we define $\hat{Q}$ by

$$
\hat{Q}\left(x_{2}, \ldots, x_{k-1}\right)=\int_{x_{1}}^{x_{k}}\left(\hat{w}^{\prime}\right)^{2} \mathrm{~d} x,
$$

where $\hat{w}$ is the solution of

$$
-\hat{w}^{\prime \prime}=\phi \operatorname{in}\left(x_{1}, x_{k}\right), \hat{w}\left(x_{1}\right)=\hat{w}\left(x_{k}\right), \hat{w}^{\prime}\left(x_{1}\right)=\hat{w}^{\prime}\left(x_{k}\right), \int_{x_{1}}^{x_{k}} \hat{w} d x=0 .
$$

Note that in the construction of $\hat{Q}$, we have deleted the $C$-macrodomain, identified the points $x_{1}$ and $x_{k}$, and solved (4.54). Clearly $\hat{Q}$ depends on $x_{2}, x_{3}, \ldots, x_{k-1}$.

Lemma 4.2. For $\mathbf{x}=\left(x_{1}, x_{2}, \ldots, x_{k}\right)$ of every $u$ in (4.32) we have $\tilde{Q}\left(x_{1}, x_{2}, \ldots, x_{k}\right) \geq \hat{Q}\left(x_{2}, x_{3}, \ldots, x_{k-1}\right)$. If $\mathbf{x}=\left(x_{1}, \ldots, x_{k}\right)$ is a critical point of $\tilde{Q}$, then $\tilde{Q}\left(x_{1}, \ldots, x_{k}\right)=\hat{Q}\left(x_{2}, \ldots, x_{k-1}\right)$.

Proof. Recall $w=b v_{\mathrm{A}}-a v_{\mathrm{B}}$ defined on $(0,1)$. Let $\hat{w}$ be given in (4.54) which is defined on $\left(x_{1}, x_{k}\right)$. Since $w^{\prime \prime}=\hat{w}^{\prime \prime}$ on $\left(x_{1}, x_{k}\right)$ there exists a constant $D$ so that $w=\hat{w}+D$ on $\left(x_{1}, x_{k}\right)$. Then 


$$
\begin{aligned}
\tilde{Q}\left(x_{1}, x_{2}, \ldots, x_{k}\right) & =\int_{0}^{1}\left(w^{\prime}\right)^{2} d x \geq \int_{x_{1}}^{x_{K}}\left(w^{\prime}\right)^{2} \mathrm{~d} x=\int_{x_{1}}^{x_{k}}\left(\hat{w}^{\prime}+D\right)^{2} \mathrm{~d} x \\
& =\int_{x_{1}}^{x_{k}}\left(\hat{w}^{\prime}\right)^{2} \mathrm{~d} x+2 D \int_{x_{1}}^{x_{k}} \hat{w}^{\prime} \mathrm{d} x+\left(x_{k}-x_{1}\right) D^{2} \\
& =\hat{Q}\left(x_{2}, \ldots, x_{k-1}\right)+(1-c) D^{2} \geq \hat{Q}\left(x_{2}, \ldots, x_{k-1}\right)
\end{aligned}
$$

since $\int_{x_{1}}^{x_{k}} \hat{w}^{\prime} \mathrm{d} x=\hat{w}\left(x_{k}\right)-\hat{w}\left(x_{1}\right)=0$.

If $\mathbf{x}$ is a critical point of $\tilde{Q}$, the argument in the proof of Lemma 4.1 shows that $w^{\prime}=0$ on the C-macrodomain. Because $\int_{0}^{1} w^{\prime} \mathrm{d} x=\int_{x_{1}}^{x_{k}} \hat{w}^{\prime} \mathrm{d} x=0$ by the periodicity of $w$ and $\hat{w}$ and $w^{\prime}=\hat{w}^{\prime}+D$ on $\left(x_{1}, x_{k}\right)$, we deduce that $w^{\prime}=\hat{w}^{\prime}$ on $\left(x_{1}, x_{k}\right)$. Therefore, $\tilde{Q}\left(x_{1}, \ldots, x_{k}\right)=\hat{Q}\left(x_{2}, \ldots, x_{k-1}\right)$.

Lemma 4.3. Any $\mathbf{x}$ given by $(4.45,4.46)$ is a strict local minimum of $\tilde{Q}$, modulo translation.

Proof. Because of Lemma 4.2, we will show that $\left(x_{2}, \ldots, x_{k-1}\right)$ of any $\mathbf{x}$ of $(4.45,4.46)$ is a strict global minimum of $\hat{Q}$ modulo translation. The lemma then follows easily.

To study $\hat{Q}$ at $\mathbf{x}$ associated with any $u$ in (4.32), we translate $\mathbf{x}$ so that $x_{1}=0$ and $x_{k}=1-c$. Then $x_{2}, x_{3}, \ldots, x_{k-1} \in(0,1-c)$ and $\hat{w}$ is defined on $(0,1-c)$. The techniques used in [25] to study a diblock copolymer system can be applied here because $\hat{Q}$ is really a diblock copolymer problem. As in the proof of Lemma 4.1

$$
\frac{\partial \hat{Q}}{\partial x_{j}}=2(-1)^{j}(a+b) \hat{w}\left(x_{j}\right), \quad j=2,3, \ldots, k-1 .
$$

Moreover, if $\left(x_{2}, \ldots, x_{k-1}\right)$ is a critical point of $\hat{Q}$, then

$$
\begin{aligned}
& 1-c-x_{k-1}+x_{2}=x_{4}-x_{3}=\cdots=x_{k-2}-x_{k-3}=\frac{2 a}{k-2}, \\
& x_{3}-x_{2}=x_{5}-x_{4}=\cdots=x_{k-1}-x_{k-2}=\frac{2 b}{k-2},
\end{aligned}
$$

and $\hat{w}^{\prime}$ is given explicitly by

$$
\hat{w}^{\prime}(x)=\left\{\begin{array}{lll}
-b\left(x-\frac{x_{2}+x_{k-1}-1+c}{2}\right) & \text { if } & x \in\left(0, x_{2}\right) \\
a\left(x-\frac{x_{2}+x_{3}}{2}\right) & \text { if } & x \in\left(x_{2}, x_{3}\right) \\
-b\left(x-\frac{x_{3}+x_{4}}{2}\right) & \text { if } & x \in\left(x_{3}, x_{4}\right) \\
\vdots & \text { if } & x \in\left(x_{k-2}, x_{k-1}\right) \\
a\left(x-\frac{x_{k-2}+x_{k-1}}{2}\right) & \text { if } & x \in\left(x_{k-1}, 1-c\right) . \\
-b\left(x-\frac{x_{k-1}+x_{2}+1-c}{2}\right) &
\end{array}\right.
$$


At this $\left(x_{2}, \ldots, x_{k-1}\right)$ we obtain from (4.56) to (4.58) that

$$
\begin{aligned}
\hat{Q}\left(x_{2}, \ldots, x_{k-1}\right) & =\int_{0}^{1-c}\left(\hat{w}^{\prime}\right)^{2} \mathrm{~d} x \\
& =\left(\frac{k}{2}-1\right)\left[a^{2} \int_{x_{2}}^{x_{3}}\left(x-\frac{x_{2}+x_{3}}{2}\right)^{2} \mathrm{~d} x+b^{2} \int_{x_{3}}^{x_{4}}\left(x-\frac{x_{3}+x_{4}}{2}\right)^{2} \mathrm{~d} x\right] \\
& =\frac{a^{2} b^{2}(a+b)}{3(k-2)^{2}} .
\end{aligned}
$$

If the global minimizer of $\hat{Q}$ is not achieved at a point given by $(4.56,4.57)$, then it must be on the boundary since every (interior) critical point of $\hat{Q}$ is given by $(4.56,4.57)$. A boundary point can be viewed as a point $\left(x_{2}^{\prime}, x_{3}^{\prime}, \ldots, x_{k^{\prime}-1}^{\prime}\right)$ for some even $k^{\prime}<k$. We are now dealing with a diblock copolymer problem with $k^{\prime}-2$ interfaces. We ask whether in this $k^{\prime}-2$ interface problem the global minimizer of $\hat{Q}$ is achieved at an interior point. If so, at such a global minimizer $\left(x_{2}^{\prime}, \ldots, x_{k^{\prime}-1}^{\prime}\right)$,

$$
\hat{Q}\left(x_{2}^{\prime}, \ldots, x_{k^{\prime}-1}^{\prime}\right)=\frac{a^{2} b^{2}(a+b)}{3\left(k^{\prime}-2\right)^{2}} .
$$

This is a contradiction because of $(4.59,4.60)$ and $\left(a^{2} b^{2}(a+b) / 3\left(k^{\prime}-2\right)^{2}\right)>\left(a^{2} b^{2}(a+b) / 3(k-2)^{2}\right)$. Otherwise we continue the induction process. Eventually we end up with the diblock copolymer problem with only two interfaces $x_{2}^{\prime \prime}$ and $x_{3}^{\prime \prime}$. There $x_{3}^{\prime \prime}-x_{2}^{\prime \prime}=b, \hat{Q}$ is constant. Moreover every $\left(x_{2}^{\prime \prime}, x_{3}^{\prime \prime}\right)$ is an interior point and is trivially a global minimizer. This proves the lemma.

We are now ready to show that the original problem $I_{\varepsilon}$ has a local minimizer which is close to the local minimizer of $Q$ given by $(4.45,4.46)$. We measure closeness by the $L^{2}\left(T^{1}\right)$ norm which is denoted by $\|\cdot\|_{2}$.

Theorem 4.4. For every positive even integer $k$ when $\varepsilon$ is small, there exists a local minimizer of $I_{\varepsilon}$ with the $C A B A B \ldots A B A$ pattern of $k$ microdomains. More precisely let $u_{0} \in(4.32)$ be given by $(4.45,4.46)$. For sufficiently small $\varepsilon$ there exists a local minimizer $u_{\varepsilon}$ of $I_{\varepsilon}$ such that

$$
\left\|u_{\varepsilon}-u_{0}\right\|_{2} \rightarrow 0 \quad \text { and } \quad \varepsilon^{-1} I_{\varepsilon}\left(u_{\varepsilon}\right) \rightarrow Q\left(u_{0}\right) \text { as } \varepsilon \rightarrow 0
$$

Theorem 4.4 is proved using the notion of $\Gamma$-convergence (cf. $[8,21,4,15])$. The proof will easily follow from the following lemmas. The domains of the functionals $I_{\varepsilon}$ and $Q$ are trivially extended to the same set

$$
\left\{u=\left(u_{\mathrm{A}}, u_{\mathrm{B}}, u_{\mathrm{C}}\right): u_{\mathrm{m}} \in L^{2}\left(T^{1}\right)(\mathrm{m}=\mathrm{A}, \mathrm{B}, \mathrm{C}), u_{\mathrm{A}}+u_{\mathrm{B}}+u_{\mathrm{C}}=1, \bar{u}_{\mathrm{A}}-a=\bar{u}_{\mathrm{B}}-b=\bar{u}_{\mathrm{C}}-c=0\right\}(4.61)
$$

by setting $I_{\varepsilon}(u)=\infty$ if $u$ is in (4.61) but not in (4.31), and $Q(u)=\infty$ if $u$ is in (4.61) but not in (4.32).

Lemma 4.5. $I_{\varepsilon} \Gamma$-converges to $Q$ in the sense that.

1. For $u_{\varepsilon}$ and $u$ in (4.61) with $\lim _{\varepsilon \rightarrow 0}\left\|u_{\varepsilon}-u\right\|_{2}=0, \liminf _{\varepsilon \rightarrow 0} \varepsilon^{-1} I_{\varepsilon}\left(u_{\varepsilon}\right) \geq Q(u)$.

2. For every $u$ in (4.61) there exist $u_{\varepsilon}$ in (4.61) such that $\lim _{\varepsilon \rightarrow 0}\left\|u_{\varepsilon}-u\right\|_{2}=0$ and $\limsup _{\varepsilon \rightarrow 0} \varepsilon^{-1} I_{\varepsilon}\left(u_{\varepsilon}\right) \leq Q(u)$.

Proof. We view $\varepsilon^{-1} I_{\varepsilon}$ as the sum of a local part and a nonlocal part. The local part is

$$
u \rightarrow \int_{0}^{1}\left[\frac{\varepsilon}{2}\left\langle K u^{\prime}, u^{\prime}\right\rangle+\frac{1}{\varepsilon} W(u)\right] \mathrm{d} x ;
$$


the nonlocal part is

$$
u \rightarrow \frac{\gamma}{2} \int_{0}^{1}\left\langle J(-\Delta)^{-1 / 2}(u-\bar{u}),(-\Delta)^{-1 / 2}(u-\bar{u})\right\rangle \mathrm{d} x
$$

It was proved in [1] that the local part $\Gamma$-converges to the functional

$$
u \longrightarrow \tau^{\mathrm{AB}} N_{\mathrm{AB}}+\tau^{\mathrm{BC}} N_{\mathrm{BC}}+\tau^{\mathrm{CA}} N_{\mathrm{CA}}
$$

which is the local part of $Q$. The nonlocal part (4.63) appears in both $I_{\varepsilon}$ and $Q$. It is considered as a continuous (with respect to the $L^{2}\left(T^{1}\right)$ norm) perturbation of the local parts $(4.62,4.64)$. It follows from the definition of $\Gamma$-convergence that the $\Gamma$-convergence property is not affected by such a perturbation. Hence $\varepsilon^{-1} I_{\varepsilon} \Gamma$-converges to $Q$.

In order to exploit the $\Gamma$-convergence, we need $\varepsilon^{-1} I_{\varepsilon}$ and $Q$ to satisfy the following compactness condition, the proof of which may be found in [25].

Lemma 4.6. Let $\varepsilon_{j}$ be a sequence of positive numbers converging to 0 , and $\left\{u_{j}\right\}$ a sequence in (4.61). If $\varepsilon_{j}^{-1} I_{\varepsilon_{j}}\left(u_{j}\right)$ is bounded above in $j$, then $\left\{u_{j}\right\}$ is relatively compact in (4.61) with respect to the $L^{2}\left(T^{1}\right)$ norm, and its cluster points belong to (4.32).

The next lemma is proved in [15]. Because of the translation invariance in our problem, the statement here is a bit different. Define a manifold of translates of $u_{0}$

$$
M\left(u_{0}\right):=\left\{u \in(4.61): u(\cdot)=u_{0}(\cdot-y), y \in T^{1}\right\}
$$

and a tube like neighborhood of $M\left(u_{0}\right)$

$$
N_{\delta}\left(u_{0}\right):=\left\{u \in(4.61):\left\|u(\cdot)-u_{0}(\cdot-y)\right\|_{2}<\delta \text {, for some } y \text { in } T^{1}\right\} .
$$

Lemma 4.7. Let $\delta>0$ and $u_{0}$ in (4.61) be such that $Q\left(u_{0}\right)<Q(u)$ for all $u \in N_{\delta}\left(u_{0}\right) \backslash M\left(u_{0}\right)$. Then there exist $\varepsilon_{0}>0$ and $u_{\varepsilon} \in N_{\delta / 2}\left(u_{0}\right)$ for all $\varepsilon<\varepsilon_{0}$ such that $I_{\varepsilon}\left(u_{\varepsilon}\right) \leq I_{\varepsilon}(u)$ for all $u \in N_{\delta / 2}\left(u_{0}\right)$. In addition under the $L^{2}\left(T^{1}\right)$ norm, $u_{\varepsilon} \rightarrow u_{0}$ up to translation as $\varepsilon \rightarrow 0$.

In Lemma 4.7 above, $Q$ is defined on (4.61). Lastly, one can show as in [25] that we may instead only consider $Q$ in each (4.33):

Lemma 4.8. If $u_{0}$ in (4.33) is a strict local minimizer, modulo translation, of $Q$ restricted to (4.33), then $u_{0}$ is also a strict local minimizer, modulo translation, of $Q$ defined in (4.61).

We are now ready to prove Theorem 4.4.

Proof of Theorem 4.4. The existence of a local minimizer $u_{\varepsilon}$ follows from Lemmas 4.3, 4.7 and 4.8. Lemma 4.7 also shows that under the $L^{2}\left(T^{1}\right)$ norm $u_{\varepsilon} \rightarrow u_{0}$ modulo translation. The convergence $\varepsilon^{-1} I_{\varepsilon}\left(u_{\varepsilon}\right) \rightarrow Q\left(u_{0}\right)$ follows from Lemma 4.5 and the fact that $u_{\varepsilon}$ minimizes $\varepsilon^{-1} I_{\varepsilon}$ in a neighborhood of $u_{0}$.

By $(4.34,4.41,4.42)$, Lemma 4.2 , and $(4.59)$, the reduced free energy of $u_{0}$ given by $(4.45,4.46)$ is

$$
Q\left(u_{0}\right)=2 \tau^{\mathrm{CA}}+(k-2) \tau^{\mathrm{AB}}+\frac{\gamma}{2} \frac{3}{2 a^{2} b^{2}} \hat{Q}\left(u_{0}\right)=2 \tau^{\mathrm{CA}}+(k-2) \tau^{\mathrm{AB}}+\frac{\gamma(a+b)}{2(k-2)^{2}} .
$$

If we minimize (4.67) with respect to $k$, we find the optimal number of $\mathrm{AB}$ interfaces:

$$
(k-2) \sim\left(\frac{\gamma(a+b)}{\tau^{\mathrm{AB}}}\right)^{1 / 3} .
$$


We conclude by noting that we are not able to characterize the global minimizers of $I_{\varepsilon}$. The $\Gamma$-convergence theory asserts that as $\varepsilon \rightarrow 0$, any global minimizer of $I_{\varepsilon}$ converges (after translation), under the $L^{2}\left(T^{1}\right)$ norm, to a global minimizer of $Q$. One would like to show that any global minimizer of $Q$ is given by $(4.45,4.46)$ with $k$ being the optimal value given in $(4.67,4.68)$. If every $\mathrm{C}$-macrodomain of a global minimizer of $Q$ is adjacent to either two A-microdomains or two B-microdomains, we may argue as in the proof of Lemma 4.1 and conclude that on each C-macrodomain $w^{\prime}=0$. It follows that the global minimizer has only one C-macrodomain and is given by $(4.45,4.46)$. Unfortunately, we have to worry about the possible appearance of ... ACB ... like combinations. As discussed in $(4.50,4.51)$ they lead to conditions that are not easily analyzed.

\section{Appendix A. General reduction to two order parameters}

Finally we mention a simple reduction to two order parameters associated with the form of the nonlocal interaction term used in [24,18]. Because of the incompressibility condition, i.e. $u_{\mathrm{A}}+u_{\mathrm{B}}+u_{\mathrm{C}}=1$, only two of the densities are required to fully describe the system. Ohta and Ito noted in [24] that the choice of ${ }^{5}$

$$
\phi=u_{\mathrm{A}}-u_{\mathrm{B}} \quad \text { and } \quad \psi=u_{\mathrm{A}}+u_{\mathrm{B}}=1-u_{\mathrm{C}},
$$

is particularly useful in that the statistics of $\psi$ capture the macrophase separation into homopolymer and copolymerrich domains, while that of $\phi$ capture the microphase separation within the copolymer-rich domains. The spatial averages $\bar{\phi}$ and $\bar{\psi}$ are some fixed number in $[-1,1]$ and $[0,1]$, respectively. Using these variables, one may readily check that the long-range interaction term (i.e. the second term in (3.22)) takes the form

$$
\int_{\Omega} \int_{\Omega} G(x, y)[\alpha(\phi(x)-\bar{\phi})(\phi(y)-\bar{\phi})+\beta(\phi(x)-\bar{\phi})(\psi(y)-\bar{\psi})+\gamma(\psi(x)-\bar{\psi})(\psi(y)-\bar{\psi})] \mathrm{d} x \mathrm{~d} y,
$$

where $G$ denotes the Green's function of the Laplacian with Neumann boundary conditions, and from (3.27), the coefficients $\alpha, \beta$ and $\gamma$ are related to the polymerization indices as follows

$$
\alpha \sim\left(\frac{1}{N_{\mathrm{A}}}+\frac{1}{N_{\mathrm{B}}}\right)^{2}, \quad \beta \sim\left(\frac{1}{N_{\mathrm{A}}^{2}}-\frac{1}{N_{\mathrm{B}}^{2}}\right), \quad \gamma \sim\left(\frac{1}{N_{\mathrm{A}}}-\frac{1}{N_{\mathrm{B}}}\right)^{2} .
$$

This agrees with the model adopted in [24,18].

\section{References}

[1] S. Baldo, Minimal interface criterion for phase transitions in mixtures of Cahn-Hilliard fluids, Ann. Inst. H. Poincaré Anal. Non Linéaire 7 (2) (1990) 67-90.

[2] R. Balian, From Microphysics to Macrophysics: Methods and Applications of Statistical Physics, Two Volumes, Springer-Verlag, Berlin, 1991.

[3] F.S. Bates, G.H. Fredrickson, Block copolymers - designer soft materials, Phys. Today 52-2 (1999) 32-38.

[4] A. Braides, Gamma Convergence for Beginners, Oxford Lecture Series in Mathematics and Its Applications, 22 (2002).

[5] J.W. Cahn, J.E. Hilliard, Free energy of a nonuniform system. I. Interfacial free energy, J. Chem. Phys. 28 (2) (1958) $258-267$.

[6] R. Choksi, X. Ren, On a derivation of a density functional theory for microphase separation of diblock copolymers, J. Stat. Phys. 113 (2003) 151-176.

\footnotetext{
${ }^{5}$ Note that these variables differ slightly than the ones used in Section 4.
} 
[7] P.G. de Gennes, Scaling Concepts in Polymer Physics, Cornell University, Ithaca, NY, 1979.

[8] E. De Giorgi, Sulla Convergenza di Alcune Successioni D'integrali del Tipo Dell'area, Rend. Mat. 8 (6) (1975) $277-294$.

[9] A.Y. Grosberg, A.R. Khokhlov, Statistical Physics of Macromolecules, American Institute of Physics (AIP) Press, New York, 1994.

[10] E. Helfand, Theory of inhomogeneous polymers: fundamentals of Gaussian random walk model, J. Chem. Phys. 62 (1975) $999-1005$.

[11] E. Helfand, Z.R. Wasserman, Block copolymer theory. 4. Narrow interphase approximations, Macromolecules 9 (1976) 879-888.

[12] E. Helfand, Z.R. Wasserman, Block copolymer theory. 5. Spherical domains, Macromolecules 11 (1978) 960.

[13] E. Helfand, Z.R. Wasserman, Block copolymer theory. 6. Cylindrical domains, Macromolecules 13 (1980) 994-998.

[14] K.M. Hong, J. Noolandi, Theory of inhomogeneous multicomponent polymer systems, Macromolecules 14 (1981) $727-736$.

[15] R.V. Kohn, P. Sternberg, Local minimisers and singular perturbations, Proc. Roy. Soc. Edinburgh Sect. A 111 (1-2) (1989) 69-84.

[16] S. Koizumi, H. Hasegawa, T. Hashimoto, Macromolecules 27 (1994) 6532.

[17] S. Koizumi, H. Hasegawa, T. Hashimoto, Macromolecules 27 (1994) 7893.

[18] A. Ito, Domain patterns in copolymer-homopolymer mixtures, Phys. Rev. E 58-5 (1998) 6158-6165.

[19] L. Leibler, Theory of microphase separation in block copolymers, Macromolecules 13 (1980) 1602-1617.

[20] M.W. Matsen, M. Schick, Stable and unstable phases of a diblock copolymer melt, Phys. Rev. Lett. 72 (1994) $2660-2663$.

[21] L. Modica, The gradient theory of phase transitions and the minimal interface criterion, Arch. Rat. Mech. Anal. 98 (2) (1987) $123-142$.

[22] Y. Nishiura, I. Ohnishi, Some mathematical aspects of the micro-phase separation in diblock copolymers, Physica D 84 (1995) 31-39.

[23] T. Ohta, K. Kawasaki, Equilibrium morphology of block copolymer melts, Macromolecules 19 (1986) 2621-2632.

[24] T. Ohta, A. Ito, Dynamics of phase separation in copolymer-homopolymer mixtures, Phys. Rev. E 52-5 (1995) 5250-5260.

[25] X. Ren, J. Wei, On the multiplicity of solutions of two nonlocal variational problems, SIAM J. Math. Anal. 31 (4) (2000) $909-924$.

[26] H. Tanaka, H. Hasegawa, T. Hashimoto, Macromolecules 24 (1991) 240. 